\title{
Animal Models of Human Granulocyte Diseases
}

\author{
Alejandro A. Schäffer ${ }^{1}$ and Christoph Klein ${ }^{2}$ \\ ${ }^{1}$ Computational Biology Branch, National Center for Biotechnology Information, National Institutes \\ of Health, Department of Health and Human Services, Bethesda, MD 20894 USA; \\ schaffer@helix.nih.gov \\ 2Department of Pediatrics, Dr. von Hauner Children's Hospital, Ludwig-maximilians-University, \\ Lindwurmstraße 4 D-80337 Munich GERMANY; Christoph.Klein@med.unimuenchen.de
}

\begin{abstract}
In vivo animal models have proven very useful to understand basic biological pathways of the immune system, a prerequisite for the development of innovate therapies. This manuscript addresses currently available models for defined human monogenetic defects of neutrophil granulocytes, including murine, zebrafish and larger mammalian species. Strengths and weaknesses of each system are summarized, and clinical investigators may thus be inspired to develop further lines of research to improve diagnosis and therapy by use of the appropriate animal model system.
\end{abstract}

\section{Keywords}

Chronic granulomatous disease; leukocyte adhesion deficiency; severe congenital neutropenia; neutrophils; mouse models; zebrafish models

\section{Introduction}

Granulocytes are immune cells that contain within them punctate granules, visible under the light microscope. There are three categories of granulocytes: basophils, neutrophils, and eosinophils that are distinguished by whether they can be usefully stained by basic (high $\mathrm{pH}$ ), neutral (medium $\mathrm{pH}$ ), or acidic (low $\mathrm{pH}$ ) stains. Eosinophils may have several roles in host defense and have been most widely studied in asthma. ${ }^{1}$ Both eosinophils and basophils have roles in promoting the Th2 responses needed to defend against parasitic infections. ${ }^{1,2}$ However, a search of OMIM (Online Mendelian Inheritance in Man) shows no monogenic disorders primarily attributed to eosinophil or basophil dysfunction. By laboratory assays, humans have been found with a partial or total lack of peroxidase in eosinophil granules, but they have mild anemia or no phenotype at all. ${ }^{3}$ Ohnmacht et al. generated mice lacking basophils, which will enable targeted studies of human diseases in which basophils may be important. ${ }^{4}$ The immune function of neutrophils and their roles in disease are much better characterized than those of basophils and eosinophils, so we focus entirely on neutrophils and their diseases.

Corresponding author: Alejandro A. Schäffer, National Center for Biotechnology Information, National Institutes of Health, 8600 Rockville Pike, Bethesda, MD 20894 USA, schaffer@helix.nih.gov, FAX: +1 301-480-2288.

Publisher's Disclaimer: This is a PDF file of an unedited manuscript that has been accepted for publication. As a service to our customers we are providing this early version of the manuscript. The manuscript will undergo copyediting, typesetting, and review of the resulting proof before it is published in its final citable form. Please note that during the production process errors may be discovered which could affect the content, and all legal disclaimers that apply to the journal pertain.

Financial disclosure and conflicts of interest: None 
Neutrophils are first responders at sites of infection and part of the innate immune system. Neutrophils can fight an infection by diverse weapons: phagocytosis of the infecting microbe,${ }^{5}$ poisoning the microbe with toxic peptides stored in the granules, ${ }^{6}$ and wrapping the microbe in neutrophil extracellular traps. ${ }^{7}$ More generally, neutrophils enhance inflammation and inflammatory signals that eventually call in macrophages, $\mathrm{T}$ cells, and B cells for a more long-lasting attack on the infection. Consistent with the paradigm that the innate immune system is more primitive than the adaptive immune system, all jawed vertebrates are thought to have neutrophils or neutrophil-like cells called heterophils, which can kill microbes by phagocytosis. ${ }^{8,9}$ Even some invertebrates, such as flies, have neutrophil-like cells. ${ }^{10}$ One reason eosinophils are thought be important for parasitic infections is that parasites, such as helminths, are too large to be killed by phagocytosis. ${ }^{1}$

Not only are neutrophils conserved across jawed vertebrates, but so are most of the known genes mutated in human neutrophil diseases. This evolutionary perspective suggests that it might be possible to model each human neutrophil disease of known monogenic cause by knocking out the corresponding gene in a mouse and characterizing the mouse phenotype. One could also hope to discover new human diseases by first studying abnormal mice that turn out to have a neutrophil disease. In a few cases, fish have been deliberately used instead of mice, and we also include naturally occurring models of two diseases in dogs and one in cows.

\section{Classification of neutrophil diseases}

Most human monogenic neutrophil diseases can be classified according to the type of malfunction. Conditions in which neutrophil defense is inadequate because the number of circulating neutrophils are too low, as measured by the absolute neutrophil count (ANC), are called neutropenia. We do not discuss chronic benign and ethnic neutropenias, but focus instead on neutropenias that may start at birth and that are associated with severe bacterial infections, called severe congenital neutropenia (SCN). Diseases in which neutrophils fail to migrate properly to sites of infection are called leukocyte adhesion deficiency (LAD). Diseases in which neutrophils fail to carry out the oxidative burst function are called chronic granulomatous disease (CGD). Based on these layman's descriptions, one might imagine that SCN would be easiest to explain at a molecular level and CGD hardest and that the causes of neutropenia would be most conserved across species and the causes of CGD least conserved. Surprisingly, the opposite is true for both measures of complexity. Therefore, we present relevant animal models in the order: CGD, then LAD, then SCN, from best understood to least understood. One rare disease, due a mutation in $R A C 2$, is at the boundary of CGD and LAD and that disease is described in the LAD section. Two monogenic diseases that do not fit into this three-part classification and some complex neutrophil diseases are described in a fourth section.

We summarize over twenty years of animal case studies to address the basic evolutionary question: For which genes does conservation of the gene imply conservation of the phenotype when the gene is mutated? From the way the question is posed, one should expect that the phenotype is conserved for some genes, but not for other genes. Clinicians may wish to know when the phenotype is conserved because for those genes and only for those genes, animal models should be useful to understand the cellular mechanism of the disease and to test treatments.

\section{Chronic Granulomatous Disease}

Chronic granulomatous diseases (CGDs) are primary immunodeficiencies characterized by recurrent infections and inflammatory lesions called granulomas. The granulomas can occur on the skin or in the liver, lymphatic system, spleen, gastrointestinal tract, or genital tract. 
Mechanistically, CGD occurs due to a defect in respiratory burst oxidase activity in neutrophils. More specifically the nicotinamide adenine dinucleotide phosphate (NADPH) oxidase complex does not produce adequate amounts of superoxide and therefore, killing of microbes is impaired.

There are five phenotypically comparable CGDs due to defects in any of five proteins that participate in the NADPH oxidase complex. These proteins are traditionally denoted according to their approximate molecular weights: p22, p40, p47, p67, gp91; the ' $\mathrm{g}$ ' in gp91 indicates that this protein is glycosylated; often, a superscript "phox" is added to all five protein names, but we omit that. Defects in a sixth participating protein RAC2 lead to a phenotype that overlaps CGD, but because RAC2 has other functions, that phenotype is more severe and is better classified as a leukocyte adhesion deficiency. The gene names are shown in Table 1.

There are documented human patients with null mutations for four out of the five types. For p40 deficiency, the only documented human patient has compound heterozygous mutations, one of which may not be null. ${ }^{13}$ The majority of human CGD patients in North America and Europe are males with a mutation in $C Y B B$, encoding gp91, because $C Y B B$ is on the $\mathrm{X}$ chromosome. The other forms of CGD are autosomal recessive and affect males and females equally.

Mice with definitely or likely functional null mutations exist for all five types. Knockout mice for $\mathrm{p} 40, \mathrm{p} 47$, and gp91 were deliberately engineered. Mice with a homozygous mutation in p67 were generated via a cross of known mouse strains. ${ }^{18}$ Mice with a null substitution mutation in p22 were created by N-ethyl-N-nitrourea (ENU) mutagenesis. ${ }^{12}$

The reported phenotypes for gp91, p47, and p40 knockout mice generally correspond to the phenotypes of the most severe human patients, with one proviso. Mouse studies typically report on immune response to challenge by a small number of microbial species, while CGD patients must cope with a plethora of infectious prokaryotes. Granulocytes of gp91-deficient male mice produce no superoxide. ${ }^{20}$ Clearance of infection by $S$. aureus and $A$. fumigatus (two infections commonly seen in CGD patients) was delayed in comparison to gp91sufficient control mice. When gp91-deficient mice were injected with the irritant chemical thioglycollate, there was increased neutrophil recruitment near the site of injection. ${ }^{20} \mathrm{p} 47$ deficient mice produce no superoxide in neutrophils. ${ }^{16}$ Despite the lack of superoxide, the knockout neutrophils have some capability to kill $S$. aureus in vitro, but it is reduced compared to p47-sufficent neutrophils. ${ }^{16}$ p47 knockout mice developed spontaneous infections and had significantly lower survival than heterozygous or wild-type mice [Jackson1995]. Histological post mortem examination identified granulomas similar to those seen in CGD patients. ${ }^{16} \mathrm{p} 40$ knockout mice have a deficiency in production of in response to TNF-alpha and their neutrophils have difficulty killing $S$. aureus (in vitro. ${ }^{14}$

The mouse model for the p67 form of CGD was obtained and characterized as follows. ${ }^{18} \mathrm{~A}$ backcross of the C57BL/6J strain (used in many laboratories) to the wild MOLF/Ei strain leads to variability in susceptibility to salmonella infections. This variation is consistent with recessive inheritance of a single locus and can be mapped to a region of mouse chromosome 1 containing the $N c f 2$ gene, encoding p67. Sequencing identified that the susceptible mice are homozygous for a R394W mutation derived from the MOLF/Ei strain. A human CGD patient with an analogous R395W mutation has been reported. A functional assay suggested that $\mathrm{R} 394 \mathrm{~W}$ leads to a deficiency in superoxide production, which is a hallmark of CGD. ${ }^{18}$

The most interesting of the CGD model mice is the one for $\mathrm{p} 22$ deficiency, which has a Y121H homozygous substitution in the protein. ${ }^{12}$ These mutant mice have the expected immunodeficiency, which in this study was demonstrated by challenge with Burkholderia, 
another infection commonly seen in CGD patients. Surprisingly, the p22 mutant mice also have a balance disorder due to faulty gravity sensing in the inner ear, which has not been reported for human patients with the p22 form of CGD. The molecular explanation for the balance defect is that there are several oxidase producing complexes that share the $\mathrm{p} 22$ subunit; these complexes use different homologs of the gp91 subunit to partner with p22. One of these complexes, called Nox3, is needed for the generation of otoconia, which are calcium carbonate crystals in the inner ear used for gravity sensing. In contrast to the balance problem, the mice have normal hearing. The Y121H substitution does not truncate the protein in principle, but in practice it leads to no (stable) protein being translated, although there is mRNA. Thus, it may be considered equivalent to a functional null for purposes of modeling.

\section{Leukocyte Adhesion Deficiency}

In this section, we cover three diseases often called "leukocyte adhesion deficiency" and a fourth one, which we here call "RAC2 disease". The three LADs are usually distinguished by Roman numerals, I, II, III. There are mouse models for each of the four diseases, and additional nonmurine animal models for two of them. For RAC2 disease, there are two zebrafish models that may be more useful than the knockout mice. ${ }^{21}$ There is one other nonmouse model for LAD type I - some Holstein cattle have a disease called bovine leukocyte adhesion deficiency (BLAD). The animal models for LAD are summarized in Table 2. LAD I, II, III have autosomal recessive inheritance; RAC2 disease is in principle autosomal dominant because the observed human mutations are heterozygous, but the observed human mutation D57N occurred de novo in the affected children ${ }^{22,23}$ and animal models suggest that biallelic null mutations of $R A C 2$ could lead to the same disease.

\section{Leukocyte adhesion deficiency, type I}

The mildest, most common and first described LAD is type I, due to biallelic mutations in the integrin gene $I T G B 2$, which codes for the protein usually called CD18. The phenotype includes recurrent bacterial infections, periodontitis, delayed wound healing and granulocytosis. ${ }^{32}$ At the molecular level, neither the integrin $\beta 1$ subunit nor its three a subunit partners come to the cell surface as they should, impairing neutrophil adhesion and chemotaxis. Two mouse models were generated ${ }^{24,25}$ that reflect a range of human LAD I phenotypes, depending on the severity of the mutations in ITGB2. A first attempt to make a knockout mouse led inadvertently to a mouse with a low level of Itgb2 expression. ${ }^{24}$ The phenotype of the mouse with the hypomorphic mutation includes: mild granulocytosis, poor neutrophil mobilization to a chemically-induced peritonitis model, and delayed rejection of heart transplants. The knockout mice have a more severe phenotype including: leukocytosis, skin infections, alopecia, defective neutrophil adhesion and migration and an impaired respiratory burst (resembling CGD). ${ }^{25}$ Knockout mice inoculated with the bacterium $S$. pneumonia have much more difficulty clearing the infection than wild-type controls and many of the mice die of infection. ${ }^{25}$

Cows with BLAD have a homozygous D128G substitution in ITGB2. The clinical symptoms overlap those of the human disease: high neutrophil counts, recurrent bacterial infections, periodontitis, oral ulcers. Leukocytes of other types appear to try to compensate for lack of innate defense by boosting IgG levels. Chemotaxis and phagocytosis of affected granulocytes are severely impaired due to poor adhesion, while adhesion-independent functions are not impaired. BLAD is of economic importance in the dairy industry because some bulls used often in mating turned out to be carriers of the BLAD-causing mutation. ${ }^{26}$ 


\section{Leukocyte adhesion deficiency, type II}

LAD II has a more severe phenotype than LAD I because it includes developmental delay and short stature. ${ }^{33}$ LAD II is caused by biallelic mutations in $S L C 35 C 1$, encoding a protein involved in transport of fucose, one of the sugars involved in post-translational protein modification. This is surprising since there is no obvious connection between fucosylation and CD18 or other integrins. The use of fucose has been partly conserved and studied in a variety of eukaryotes and prokaryotes. Indeed, one of the two seminal human studies reasoned that fucosylation pathway genes are conserved between humans and worms and used a complementation strategy in $C$. elegans to find that biallelic mutations of $S L C 35 C 1$ cause LAD II. ${ }^{34}$

For LAD II, Hellbusch et al. engineered mice that are homozygous null for $51 \mathrm{c} 35 \mathrm{c} 1$, which encodes the ortholog of the protein mutated in human LAD II. ${ }^{27}$ Mice are born in 1/2/1 proportions, so there is no prenatal lethality. The knockout mice have leukocytosis, especially elevated neutrophils and eosinophils and poor post-natal survival. The mouse weights are reduced. Leukocyte rolling flux fractions and velocity are significantly reduced, perfectly consistent with the human LAD II phenotype.

In addition to obtaining these phenotypic characterizations, Hellbusch et al. used the Slc5c1-/- mice to gain insight into the pathways of fucose transport. ${ }^{27}$ AAL (A. aurantia lectin) is specific for three types of fucosylation and can be used in vitro to show that SLc35c1-/- MEFs have a defect in a fucosylation pathway. It was known from previous work that there are two pathways to obtain GDP-fucose for fucosylation: a de novo synthesis pathway and a salvage pathway. Fucose is needed in the ER and in the Golgi; the set of fucosyltransferases in those two organelles are non-overlapping. Slc35c1 transports GDB-fucose only into the Golgi, not the ER. Yet, the mouse phenotype can be improved (better weight and survival) by administration of fucose, which must somehow get transported to the Golgi. ${ }^{27}$ This suggests that there is an alternative mechanism of transport that is less efficient, but works better as the concentration of fucose increases. Administration of fucose to one human LAD II patient similarly improved the patient's condition, but for a different patient the fucose treatment was ineffective. ${ }^{33}$ Further mouse studies, perhaps using mice in which two fucose pathways are disrupted, could give insight into the function of $\mathrm{Slc5cl}$ and its dysfunction in LAD II.

\section{Leukocyte adhesion deficiency, type III}

LAD III is a severe form of LAD that combines the immunodeficiency of LAD I with a severe bleeding disorder due to faulty platelet function. LAD III is caused by biallelic mutations in FERMT3, which encodes the protein kindlin $3 .{ }^{35-37}$ The bleeding can be more problematic clinically than the immunodeficiency. ${ }^{36}$ Analogously, Fermt 3 knockout mice die shortly after birth due to the platelet disorder, and the initial characterization of these mice does not consider granulocytes. ${ }^{28}$ To address this limitation, Moser et al. went on to generate Fermt3-/- fetal liver chimeras and neutrophils. ${ }^{29}$ Using these cells, they could show that the neutrophils have an adhesion deficiency. ${ }^{29}$ They suggest that the granulocytosis in LAD III (and by inference in LAD I) appears to be a compensation for the inability of the granulocytes to adhere and migrate to infected sites. ${ }^{29}$ They connected LAD III to LAD I at the molecular level by showing that kindlin 3 binds to integrin $\beta_{2}$ and they did integrin mutagenesis experiments to show that the NXXF motif at positions 764-767 is needed for kindlin-integrin binding. ${ }^{29}$ Although LAD III is fatal if untreated, it can be cured with hematopoietic stem cell transplant (HSCT) in some cases. ${ }^{35,36}$ because FERMT3, unlike other kindlin-encoding genes, is expressed only in cells of the hematopoietic lineage. ${ }^{28,37}$ One difference between the human LAD III phenotype and the knockout mouse 
phenotype is that human patient erythrocytes have a normal shape, but knockout mouse erythrocytes have an aberrant shape. ${ }^{36}$

\section{RAC2 disease}

RAC2 disease is functionally related to LAD and has been reported in two human patients, both carrying de novo heterozygous D57N mutations. ${ }^{22,23,38}$ The phenotype includes recurrent infections, abscesses, impaired wound healing, and lack of pus. The protein is translated at half the normal level, but the D57N mutation has a dominant negative functional effect. In vitro, the patient neutrophils were deficient in polarization, chemotaxis, azurophilic (but not specific) granule release, and superoxide anion production. ${ }^{22}$ The first patient was treated successfully with HSCT via donation from a sibling. ${ }^{38}$ The HSCT process ablated the original bone marrow, so that post-transplant, the cells are $100 \%$ donorderived. This stringency is important since D57N is a dominant-negative substitution.

RAC2 disease overlaps CGD phenotypically because of the role of RAC2 in the NADPH complex, explained in the previous section. The disease is more severe because RAC2 has roles in cell adhesion, cell migration and other pathways. ${ }^{31}$ Clarifying RAC2 functions and how they are disrupted in RAC2 disease is difficult because in mammals, there are three paralogous members of the Rac subfamily of Rho GTPases that overlap in functionality and differ primarily by tissue of expression. ${ }^{31}$ Rac1 is ubiquitously expressed; Rac2 is expressed solely in cells of the hematopoietic lineage, and Rac3 is expressed mostly in the brain. ${ }^{31}$

Roberts et al. made Rac2- $/$ - mice and Gu et al. engineered various single and double gene knockout mice for the Rac genes. ${ }^{30,31}$ They showed that Rac2-deficient cells have a defect in adhesion to fibronectin. ${ }^{31}$ The mice do not suffer from opportunistic infections, but are susceptible to $P$. aeruginosa infection, if challenged.$^{30}$ Rac2-/- mice have leukocytosis mostly due to a three-fold increase in the ANC. ${ }^{30}$ Superoxide production is impaired in vitro, but in vivo it seems that Rac1 can substitute for Rac2 in stimulating superoxide production; ${ }^{30}$ in the superoxide aspect of the phenotype, Rac 2 deficiency matches CGD rather than LAD. Neutrophil chemotaxis is impaired and Rac2-deficient neutrophils had impaired spreading on surfaces after integrin ligation, which explains the similarity of Rac2 deficiency to LAD I. ${ }^{30}$ Rac2-deficient cells also have a significant reduction in proliferation and a significant increase in apoptosis. Rac2-deficient cells have a defect in actin polymerization and they do not migrate in response to SDF1 signaling.

Recently, Deng et al. developed zebrafish models for human RAC2 disease. ${ }^{21}$ First, they prepared a morpholino with impaired Rac2 expression; second, they engineered a knock-in of the human D57N mutation. Both fish models show similar aberrant phenotypes, strengthening the evidence that D57N causes disease by a dominant-negative mechanism rather than haploinsufficienciency. Either expression of Rac2D57Nor the morpholino model causes a failure in wound healing due to impaired neutrophil chemotaxis. In both models, neutrophils fail to respond to a $P$. aeruginosa infection. Both models show a defect in polarization, which can be visualized due to the transparent nature of the zebrafish. Both models show neutrophilia without increased neutrophil production, consistent with the human phenotype. Even more interesting, the Rac2D57N mutant was used to study a neutropenia syndrome, called WHIM syndrome, as described in the next section. ${ }^{39}$

\section{Severe Congenital Neutropenia}

In severe congenital neutropenia ( $\mathrm{SCN}$ ) there is a primary immunodeficiency because the number of neutrophils in circulation is too low to fight infections adequately. In humans, an ANC below 500/ $\mu 1$, when not infected or under G-CSF (granulocyte colony stimulating factor) treatment, is considered neutropenic; some authors use a lower threshold of 200 
when adding the adjective "severe". ${ }^{40}$ Recombinant G-CSF boosts the ANC in most SCN patients ${ }^{41}$ and some untreated $\mathrm{SCN}$ patients mount a partial response to infections leading to temporarily higher ANC. The receptor for G-CSF is encoded by the gene CSF3R, and as one might expect knocking out the corresponding gene in mice leads to neutropenia (Table 3). ${ }^{42}$ Therefore, it is surprising that no SCN patients have been reported with biallelic inactivating mutations of $C S F 3 R$. Instead, some somatic mutations have been reported in patients undergoing G-CSF treatment. That inactivating mutations of CSF3R should lead to neutropenia is supported by the surprising discovery of a family in which the phenotype of neutrophilia is associated with a heterozygous $\mathrm{T} 617 \mathrm{~N}$ mutation that leads to a constitutively active form of the receptor. ${ }^{43}$

In the Introduction, we made the traditional distinction between defects of neutrophil function (CGD and LAD) and defects of neutrophil number (SCN), but this is an oversimplification. In some types of SCN, e.g., Hermansky-Pudlak syndrome type 2 (HPS2), those neutrophils that do circulate have a defective phagocytosis capability, ${ }^{44}$ but this is not a necessary or sufficient condition for a diagnosis of SCN. Other qualitative defects of neutrophil granulocytes, such as reduced calcium flux, ${ }^{45}$ may also be associated with SCN and contribute to the severity of the infections.

\section{$\mathrm{SCN}$ is heterogeneous in multiple ways}

Animal models for SCN known in 2006 were reviewed previously, ${ }^{46}$ so we focus on more recent results. Neutrophils are among the most primitive immune cells, but a surprising number of genes and pathways seem to interact to determine the ANC. ${ }^{46,47}$ The heterogeneity manifests at several levels. At the level of inheritance, SCN can be dominant (e.g., due to mutation in ELANE, GFII), recessive (e.g., HAX1, G6PC3) or X-linked (e.g., $W A S, T A Z$ ). At the level of phenotype, it had been traditional through approximately 2007 to distinguish between syndromic forms of SCN and non-syndromic forms. Classical syndromic forms that have animal models include: Hermansky-Pudlak syndrome type 2 (HPS2) due to mutations in $A P 3 B 1$, Griscelli syndrome type 2 (GS2) due to mutations in $R A B 27 A$, Chediak-Higashi syndrome (CHS) due to mutations in $L Y S T$, Cohen syndrome due to mutations in VPS13B, WHIM syndrome due to truncating mutations in $C X C R 4$, and Barth syndrome due to mutations in $T A Z$ (Tables 3 and 4). However, as indicated in Table 3 , the knockout mouse models for HPS2, GS2, and CHS are not known to have an inherent defect in neutrophil number or function. In all three cases, hypopigmentation is a more visible part of the phenotype and has received most of the attention. In the case of GS2, Schmid et al. showed that Rab27a-/- mice are neutropenic when challenged with a viral infection; this is in line with the observation that GS2 patients may have intermittent neutropenia, especially coincident with viral infections. ${ }^{48}$

The utility of the distinction between syndromic and nonsyndromic forms has been diminished since 2007 because it was discovered that in humans, biallelic $H A X 1$ mutations $^{68}$ or biallelic G6PC3 mutations ${ }^{69}$ can cause either syndromic or non-syndromic neutropenia. There are mouse models for both forms of SCN, but the relevance to the nonneutrophil symptoms differs. G6pc3-/- mice are neutropenic ${ }^{63}$ but do not share the cardiac defects and urogenital defects and other dysmorphologies seen in some humans with G6PC3 deficiency. In contrast, Hax 1-deficient mice ${ }^{64}$ share the neurological deficiencies of the HAX1-deficient patients, ${ }^{68,70,71}$ but do not accurately model HAX1-deficient patients whose phenotype is limited to neutropenia. ${ }^{71}$

\section{The newest animal models for SCN}

The recently characterized dog model for Cohen syndrome, ${ }^{67}$ called "trapped neutrophil syndrome" (TNS) in the border collie breed, provides an example of the limitations of 
modeling complex human syndromes in inbred animals. Unfortunately for breeders of border collies, the carrier frequency of the four base-pair deletion causing TNS is high (estimated at 0.04-0.08). ${ }^{67}$ Fortunately for SCN epidemiology, this high carrier frequency makes it possible to identify many border collies that are homozygous for the TNS-causing mutation, but would not necessarily be brought to the attention of a veterinarian. Shearman and Wilton showed (their Table 3 ) that human Cohen syndrome patients ${ }^{72}$ and border collies with biallelic mutations in VPS13B have variable phenotypes, despite the shared mutation and the highly inbred history of the dogs. Two differences are striking: 1) all the dogs have TNS, but not all human Cohen syndrome patients have SCN; 2) essentially all human patients have developmental delay, but fewer than half the dogs have developmental delay. $67,73,74$

The zebrafish model of WHIM syndrome ${ }^{39}$ (Table 4) is special because it enabled a proof of the unexpected result that the neutrophil aspects of RAC2 disease and WHIM syndrome are complementary. The ' $\mathrm{M}$ ' in WHIM stands for myelokathexis meaning that the ANC is low because neutrophils are stuck in the bone marrow. In contrast, most other forms of SCN are due to excessive apoptosis of neutrophils that are not necessarily stuck in the marrow. Two diseases where granulopoiesis is defective are SCN due to GFI1 heterozygous mutations ${ }^{60,75}$ and the syndrome reticular dysgenesis discussed in the next section. WHIM syndrome is caused by heterozygous, truncating mutations of the chemokine receptor $C X C R 4^{76}$ for which zebrafish have a homolog. Walters et al. showed that transgenic expression of a human mutation in zebrafish embryos does lead to neutrophils being trapped. ${ }^{39}$ They went on to show that expressing the deleterious D57N RAC2 mutation has the surprising, beneficial effect of getting the neutrophils to move properly again. Thus, RAC2 disease and WHIM syndrome demonstrate an interaction between neutrophil related proteins, which is an aspect of complex diseases that are covered in the next section.

\section{Additional discrepancies between human SCN and mouse models}

Unlike CGD and LAD, there are discrepancies among the genes known to cause recessive neutropenia in human patients and knockout mice. In addition to the "No" entries in the rightmost column of Table 3, biallelic mutations in $R M R P^{77}$ and $C 16$ orf $57^{78}$ cause syndromic forms of recessive neutropenia in humans, but mouse models have not been documented. Mice with either $B c 12 a 1^{79}$ or $M c l 1^{80}$ knocked out (the MCL1 model is a conditional knockout) have phenotypes that include neutropenia, but no humans having biallelic mutations in the orthologous $B C L 2 A 1$ or $M C L 1$ have been described. Csf3 and Csf3r are listed in Table 3 and have the same mouse vs. human discrepancy. The prospect that new mouse models may facilitate the discovery of human SCN genes is a focus of the Discussion.

\section{Other Granulocyte Diseases and Complex Diseases}

In this section, we cover two human monogenic diseases, CTSC deficiency and AK2 deficiency, which have animal models, but do not fit easily into any of the three preceding sections. This leads naturally to some mention of complex diseases, where CTSC and p47 animal models have been characterized.

The first miscellaneous monogenic disease is called reticular dysgenesis or adenylate kinase 2 (AK2) deficiency. AK2 deficiency is the most severe variant of SCID (severe combined immunodeficiency disorder) characterized by extreme paucity of T- and B-cells and neutrophils. ${ }^{81,82}$. Reticular dysgenesis is syndromic because it also includes deafness as part of the human phenotype. The immune part of the phenotype can be cured with HSCT shortly after birth. ${ }^{81}$ Pannicke et al. generated a zebrafish model in which the orthologous gene $a k 2$ was inactivated by a splice site morpholino, but the primary analysis of the fish 
was of lymphocytes, not granulocytes. ${ }^{82}$ The fish lymphocytes do not develop, as expected from the human phenotype. ${ }^{82}$ Although adenyl kinase activity is needed in most if not all cells, the phenotype of AK2 deficiency is specific to the immune system and the ear because in these cells, the paralogous AK1 cannot substitute for the non-functional AK2. ${ }^{82}$

The second miscellaneous monogenic disease is a disease of aberrant neutrophil function. Papillon-Lefèvre syndrome (PLS) is caused by biallelic mutations in cathepsin C (current gene symbol $C T S C$, previously represented by the gene symbol DPPI). ${ }^{83,84}$ The main symptoms of PLS are: 1) severe periodontal disease and loss of teeth due to infections and 2) keratosis affecting hands knees and feet. CTSC is involved in activation of granulocyte serine proteases. The engineering of $C t s c$ knockout mice was described months before the publication of the first human mutations, but the study focused on non-immune aspects of the mouse phenotype ${ }^{85}$ CTSC has a defined function in neutrophil killing of microbes and functional studies have shown roles for CTSC in decreased neutrophil chemotaxis, impaired neutrophil phagocytosis, and defective superoxide production. ${ }^{83}$ Therefore, it is surprising that only some PLS patients are susceptible to infections. ${ }^{83}$ Years later, it remains unclear whether the immune aspects of the PLS phenotype are associated with the specific mutation in $C T S C$, modifier genes, or non-genetic factors.

Although the Ctsc knockout mice were not directly helpful in characterizing PLS, they have been useful studying complex inflammatory disease. Mice lacking $C t s c$ have a significantly

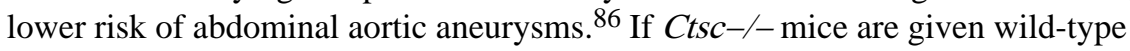
neutrophils, the susceptibility is restored, showing that the aneurysms are due in part to the inflammation produced by neutrophil recruitment. The neutrophil inflammation leading to aneurysms is associated with increased expression of $\mathrm{Cxcl} 2$, whose expression is low in Ctsc $-/$ mice but becomes high when these mice are provided with wild-type neutrophils.

\section{Mouse models pertinent to complex neutrophil diseases}

Two other studies of complex diseases and one of the genes/proteins mentioned above concern p47 (a CGD protein from Table 1).Two mouse strains that have been studied for decades for the phenotypes of diabetes and obesity turn out to be homozygous for a mutation in p47 that eliminates superoxide production. ${ }^{87}$ However, the mice were apparently not tested for immunodeficiency by microbial challenge. The variation in the diabetes and obesity phenotypes had been previously attributed to a mutation in the leptin receptor, which is on mouse chromosome 4 . Interestingly, $N c f 1$, which encodes p 47 , is located on mouse chromosome 5, but it seems that the mutations cosegregate due to a combination of randomness and the structured nature of mouse breeding crosses. Whether the Ncf1 mutation is relevant to the diabetes should be investigated by further crosses that unlink these two mutations on different chromosomes.

The mouse mutation of $\mathrm{p} 47$ was crossed into a different strain of mice susceptible to arthritis and leads to increased susceptibility to arthritis in that model. ${ }^{88}$ This study also showed that the p47 mutation in the leptin receptor deficient mouse leads to expression and translation of a truncated protein. The authors speculate that this abnormal protein may have some role in promoting autoimmune disease. ${ }^{88}$

\section{Discussion}

Our review of animal models for granulocyte deficiencies gives a mixed picture. Most models for human monogenic granulocyte diseases are based on mice, usually engineered knockout mice. Sometimes the animal model phenotype mimics the human disease phenotype and sometimes not. In most cases, the discovery of the human gene has preceded a targeted attempt to create animal models. One notable exception is G6pc3-/- mice, ${ }^{63}$ 
which were generated before the identification of any human G6PC3-deficient patients and highlighted $G 6 P C 3$ as a functional candidate located in the genetic linkage interval for the first multiplex pedigree characterized with G6PC3 deficiency. ${ }^{69}$ In contrast, Ctsc-/- mice ${ }^{85}$ were described a few months before the discovery that CTSC mutations cause human PLS, ${ }^{83,84}$ but the mouse study makes no mention of any skin or tooth phenotype. ${ }^{85} \mathrm{~A}$ recent study goes further to show that Ctsc-/- mice are not especially sensitive to Aspergillus infections. ${ }^{89}$ Surprisingly, there are only five diseases covered in this review for which the characterization of the mouse model preceded the discovery of the first human mutation; the other three are: the p40 form of CGD, LAD III, and RAC2 disease.

The contrast between the success with $G 6 p c 3$ and lack of success with $C t s c$ exemplifies the mixed picture. In the remainder of this Discussion, we take the approach of "first the good news" by summarizing recent positive developments. Then, we continue with "the bad news" of gene-specific open problems. We end with some opportunities to use animal models for neutrophil diseases that to date, have been largely missed opportunities. Perhaps, a clear delineation of open problems and open opportunities can steer future research towards the ultimate goal of making animals more relevant to the diagnosis and treatment of human patients with neutrophil diseases.

One of the more promising indications of progress in animal models is the recent development of zebrafish models, such as those for WHIM syndrome ${ }^{39}$ and RAC2 disease. ${ }^{21}$ Using zebrafish embryos, researchers can directly visualize the normal and aberrant functions of neutrophils. ${ }^{90}$ The point that it is less expensive to house zebrafish, feed zebrafish, and mutate the genomes of zebrafish than it is for mice makes zebrafish models especially attractive. One opportunity that has been so far missed in this direction is analysis of the neutrophils in a zebrafish model of Barth syndrome, ${ }^{91}$ for which other nonimmune aspects of the phenotype were evaluated. In a few cases, such as Barth syndrome or LAD II, it may be possible to use invertebrate models, but this can be tricky since it is often the case that there is not a clear one-to-one orthology relationship among sets of homologous invertebrate and human genes.

The opportunity to study complex, polygenic diseases is perhaps the most positive recent development in the area of animal models for neutrophil diseases. We mentioned in the previous section the interesting progress on CTSC and p47 in complex diseases. However, a similar opportunity to study p67 in lupus and inflammatory bowel disease has been missed, so far. A recent study showed a strong association between the H389Q amino acid substitution and human lupus, especially childhood onset. ${ }^{92}$ The H389Q variant leads to reduced superoxide production, although most lupus patients with this variant do not meet the diagnostic criteria for CGD. Another recent study showed that the R38Q amino acid substitution is associated with early-onset inflammatory bowel disease; this variant affects the interaction between p67 and RAC2. ${ }^{93}$ The findings that mutations of two different members of the NADPH complex are associated with autoimmunity in mice ${ }^{88}$ and humans ${ }^{92,93}$ suggest further studies of the other members of the complex in autoimmune diseases. The distinction in the type of autoimmune disease is at least partly due to the $\mathrm{p} 47$ mouse mutation being backcrossed onto an arthritis-prone strain. ${ }^{88}$

\section{Some open problems}

The catalog of animal models for monogenic granulocyte diseases (Tables 1,2,3,4) is extensive, but incomplete. Open problems about specific diseases include:

1. Are there any human patients with biallelic mutations in $R A C 2$ ? If so, what is the phenotype? 
2. Are there any human patients with the p40phox form of CGD and biallelic null mutations?

3. Why do human patients with the p22phox form of CGD not have problems with their balance? ${ }^{11,12}$

4. Why do many G6PC3-deficient humans have heart defects and urogenital defects, while G6pc3-deficient mice have non-syndromic neutropenia?

5. Why do pearl mice and ashen mice have no defect in neutrophil number or function?

6. Why do Elane knockin mice have normal neutrophil counts, unless endoplasmic reticulum stress is chemically induced $?^{54}$

7. If neutropenia-causing heterozygous human mutations in GFI1 are knocked in to a live mouse, would the mouse be neutropenic as suggested by the cell culture experiments of Zarebski et al. ${ }^{60}$ ?

8. Is it possible to construct a model organism for HAX1 deficiency that has neutropenia, but no neurological phenotype?

9. Is it possible to construct a knock-in mouse or fish model of the p67phox variants recently implicated in susceptibility to lupus or inflammatory bowel disease $?^{92,93}$

\section{What about ENU mutagenesisas a method to construct new animal models?}

Generalizing beyond single diseases, we observe there have been very few examples to illustrate two important opportunities of animal models of granulocyte diseases that are seen repeatedly for other diseases, including other immune diseases. These are the use of ENU mutagenesis to generate mutant mice and the use of animal models in pharmacological experiments. Hundreds of mouse models for human monogenic diseases, including dozens of immune diseases have been generated by ENU mutagenesis. The laboratories of Bruce Beutler (2011 Nobel Prize winner in Physiology or Medicine) and Chris Goodnow are renowned for ENU-generated mouse models of immune phenotypes. ${ }^{94,95}$ Another massive ENU mutagenesis screen, including but not limited to immune phenotypes, was done in the lab of Martin Hrabé de Angelis. ${ }^{96}$ Therefore, it is disappointing to observe that the only ENU-derived models listed above are for the p22phox form of CGD, ${ }^{12}$ for $\mathrm{CHS}^{51}$ and HPS2. ${ }^{56}$ In the last two models (grey mice and bullet gray mice), the number and function of the mouse neutrophils have not been characterized.

A subtle advantage of ENU mutagenesis is that the mutated genes seem to be targeted at random. Therefore, there exists the possibility to characterize a monogenic disease in a mouse before the analogous human disease is identified and without much prior understanding of the gene's normal function. However, this did not occur for p22phox, since humans with that form of CGD were described ${ }^{11}$ before the mutant mice were generated. ${ }^{12}$ Another advantage of ENU mutagenesis is that ENU mutates single nucleotides and as a consequence, in many cases, the mutant gene can be translated to a full-length protein of aberrant function. These proteins with substitution mutations can lead to phenotypes that are milder, later onset, or even different from the phenotype of mice with the same gene/protein knocked out. Moreover, the specific substitution mutations observed in the ENU-generated mice can give insight into which parts of the protein are important for its function.

The prospects for ENU mutagenesis would seem to be bright for discovering new, monogenic forms of SCN because this disease has surprising locus heterogeneity ${ }^{46}$ and can be defined by a well-established laboratory assay (ANC). ${ }^{40}$ Progress in identifying new monogenic forms of SCN is slow, with only HAX1 deficiency 68 and G6PC3 deficiency 69 
and $\mathrm{C} 16$ orf57 deficiency ${ }^{78}$ found in the past five years. Among these three genes, only $H A X 1$ and $G 6 P C 3$ could explain some nonsyndromic cases, and the large majority of patients with nonsyndromic SCN sequenced for $H A X 1$ and $G 6 P C 3$ do not have mutations. ${ }^{69,97,98}$ The approach of using human GWAS (genome-wide association studies) of variation of ANC has failed in the sense that loci near plausible candidate genes can be found to account for a small portion of the variation in ANC and those statistical associations can be replicated, ${ }^{47}$ but to date, the genes identified by GWAS have not been found to be mutated in human SCN patients.

\title{
Use of animal models to test potential therapies
}

Since this review appears in a volume concerning clinical hematology, we close with this challenge: How can animal models of neutrophil diseases be used to develop new and better treatments for patients? SCN patients can be treated with G-CSF for the neutropenia, but the mortality from infections, leukemia and other conditions remains high. ${ }^{99}$ Among the diseases we covered, the current prospects for patients with LAD III and CHS are especially grim. As high-throughput drug screens and targeted gene therapy become increasingly used in general, one can hope that these technologies can be tested on animal models on the path to better treatments for human patients.

In the early years of this field, a mouse model for the p47 form of CGD was used to show the feasibility of somatic gene therapy. ${ }^{100}$ This model and the gp91 model were used to investigate the mechanism by which interferon-gamma may be a useful treatment for CGD. ${ }^{101,102}$ This is an important question, since the use of interferon-gamma has been controversial, but the studies in mice came only after human clinical trials. Sadly, we could not find evidence that any of the monogenic animal models listed above has been used to test any drugs for that disease. The closest example is the relevance of $C t s c-/-$ mice to clinical trials of CTSC inhibitor drugs to inflammatory diseases [reviewed in reference 103]. Astute clinicians can be aware that if the dental, skin, or immune aspects of PLS start to appear, then CTSC is probably be inhibited more than is advisable.

The idea to use animal models to study the function of neutrophils or neutrophil-like cells is not new. Paul Ehrlich, co-winner of the Nobel Prize in Physiology or Medicine in 1908, discovered the stains that could distinguish neutrophils, basophils, and eosinophils under the light microscope. Ehrlich shared the prize with Ilya Mechnikov, who discovered phagocytosis in marine invertebrates. More than one hundred years later, opportunities abound to use animal models in mice, zebrafish and other species, to learn more about phagocytic neutrophils and their aberrations in human patients.

\section{Acknowledgments}

\author{
Funding: \\ The research of A.A.S. is supported by the Intramural Research program of the NIH, NLM. \\ The neutropenia research of C. K. is supported by grants from the Deutsche Forschungsgemeinschaft (DFG \\ Gottfried-Wilhelm Leibniz Program), the European Research Council (ERC Advanced Grant), the \\ Bundesministerium für Bildung und Forschung, and the Care-for-Rare Foundation.
}

\section{References}

1. Kita H. Eosinophils: multifaceted biological properties and roles in health and disease. Immunol Rev. 2011; 242:161-177. [PubMed: 21682744]

2. Karasuyama H, Mukai K, Obata K, et al. Nonredundant roles of basophils in immunity. Annu Rev Immunol. 2011; 29:45-69. [PubMed: 21166539] 
3. Lepelley P, Zandecky M, Parquet S, et al. Total peroxidase deficiency in eosinophils: a report on twin sisters, one with a refractory anaemia. Europ J Haemat. 1987; 39:77-81.

4. Ohnmacht C, Schwartz C, Panzer M, et al. Basophils orchestrate chronic allergic dermatitis and protective immunity against helminths. Immunity. 2010; 33:364-374. [PubMed: 20817571]

5. Dale DC, Boxer L, Liles WC. The phagocytes: neutrophils and monocytes. Blood. 2008; 112:935945. [PubMed: 18684880]

6. Segal AW. How neutrophils kill microbes. Ann Rev Immunol. 2005; 23:197-223. [PubMed: 15771570]

7. Fuchs TA, Abed U, Goosmann C, et al. Novel cell death program leads to neutrophil extracellular traps. J Cell Biol. 2007; 176:231-241. [PubMed: 17210947]

8. Styrt B. Species variation in neutrophil biochemistry and function. J Leuk Biol. 1989; 46:63-74.

9. Lieschke GJ, Trede NS. Fish immunology. Curr Biol. 2009; 19:R678-R682. [PubMed: 19706273]

10. Qiu P, Pan PC, Govind S. A role for the Drosophila Toll/Cactus pathway in larval hematopoiesis. Development. 1998; 125:1909-1920. [PubMed: 9550723]

11. Dinauer MC, Pierce EA, Bruns GAP, et al. Human neutrophil cytochrome b light chain (p22phox): gene structure, chromosomal location, and mutations in cytochrome-negative autosomal recessive chronic granulomatous disease. J Clin Invest. 1990; 86:1729-1737. [PubMed: 2243141]

12. Nakano Y, Longo-Guess CM, Bergstrom DE, et al. Mutation of the Cyba gene encoding p22phox causes vestibular and immune defects in mice. J Clin Invest. 2008; 118:1176-1185. [PubMed: 18292807]

13. Matute JD, Arias AA, Wright NAM, et al. A new genetic subgroup of chronic granulomatous disease with autosomal recessive mutations in $\mathrm{p} 40^{\text {phox }}$ and selective deficits in neutrophil NADPH oxidase activity. Blood. 2009; 114:3309-3315. [PubMed: 19692703]

14. Ellson CD, Davidson K, Ferguson GJ, et al. Neutrophils from p40phox-/- mice exhibit severe defects in NADPH oxidase regulation and oxidant-dependent bacterial killing. J Exp Med. 2006:1927-1937. [PubMed: 16880254]

15. Casimir CM, Bu-Ghanim HN, Rodaway ARF, et al. Autosomal recessive chronic granulomatous disease caused by deletion at a dinucleotide repeat. Proc Natl Acad Sci USA. 1991; 88:2753-2757. [PubMed: 2011585]

16. Jackson SH, Gallin JI, Holland SM. The p47phox mouse knock-out model of chronic granulomatous disease. J Exp Med. 1995; 182:751-758. [PubMed: 7650482]

17. Nunoi H, Iwata M, Tatsuzawa S, et al. AG dinucleotide insertion in a patient with chronic granulomatous disease lacking cytosolic 67-kD protein. Blood. 1995; 86:329-333. [PubMed: 7795241]

18. Sancho-Shimizu V, Malo D. Sequencing, expression, and functional analyses support the candidacy of Ncf2 in susceptibility to Salmonella typhimurium infection in wild-derived mice. J Immunol. 2006; 176:6954-6961. [PubMed: 16709856]

19. Dinauer MC, Orkin SH, Brown R, et al. The glycoprotein encoded by the X-linked chronic granulomatous disease locus is a component of the neutrophil cytochrome $b$ complex. Nature. 1987; 327:717-720. [PubMed: 3600768]

20. Pollock JD, Williams DA, Gifford MAC, et al. Mouse model of X-linked chronic granulomatous disease, an inherited defect in phagocyte superoxide production. Nature Genet. 1995; 9:202-208. [PubMed: 7719350]

21. Deng Q, Yoo SK, Cavnar PJ, et al. Dual roles for Rac2 in neutrophil motility and active retention in zebrafish hematopoietic tissue. Dev Cell. 2011; 21:735-745. [PubMed: 22014524]

22. Ambruso DR, Knall C, Abell AN, et al. Human neutrophil immunodeficiency syndrome is associated with an inhibitory Rac2 mutation. Proc Natl Acad Sci USA. 2000; 97:4654-4659. [PubMed: 10758162]

23. Accetta D, Syverson G, Bonacci B, et al. Human phagocyte defect caused by a Rac2 mutation detected by means of neonatal screening for T-cell lymphopenia. J Allergy Clin Immunol. 2011; 127:535.e2-538.e2. [PubMed: 21167572]

24. Wilson RW, Ballantyne CM, Smith CW, et al. Gene targeting yields a CD18-mutant mouse for study of inflammation. J Immunol. 1993; 151:1571-1578. [PubMed: 8101543] 
25. Scharffetter-Kochanek K, Lu H, et al. Spontaneous skin ulceration and defective T cell function in CD18 null mice. J Exp Med. 1998; 188:119-131. [PubMed: 9653089]

26. Nagahata H. Bovine leukocyte adhesion deficiency (BLAD): A Review. J Vet Med Sci. 2004; 66:1475-1482. [PubMed: 15644595]

27. Hellbusch CC, Sperandio M, Frommhold D, et al. Golgi GDP-fucose transporter-deficient mice mimic congenital disorder of glycosylation IIc/leukocyte adhesion deficiency II. J Biol Chem. 2007; 282:10762-10772. [PubMed: 17276979]

28. Moser M, Nieswandt B, Ussar S, et al. Kindlin-3 is essential for integrin activation and platelet aggregation. Nature Med. 2008; 14:325-330. [PubMed: 18278053]

29. Moser M, Bauer M, Schmid S, et al. Kindlin-3 is required for $\beta-2$ integrin-mediated leukocyte adhesion to endothelial cells. Nature Med. 2009; 15:300-305. [PubMed: 19234461]

30. Roberts AW, Kim C, Zhen L, et al. Deficiency of the hematopoietic cell-specific Rho family GTPase Rac2 is characterized by abnormalities in neutrophil function and host defense. Immunity. 1999; 10:183-196. [PubMed: 10072071]

31. Gu Y, Filippi M-D, Cancelas JA, et al. Hematopoietic cell regulation by Rac1 and Rac2 guanosine triphosphatases. Science. 2003; 302:445-449. [PubMed: 14564009]

32. Springer TA, Thompson WS, Miller LJ, et al. Inherited deficiency of the Mac-1, LFA-1, p150,95 glycoprotein family and its molecular basis. J Exp Med. 1984; 160:1901-1918. [PubMed: 6096477]

33. Lübke T, Marquardt T, Etzioni A, et al. Complementation cloning identifies CDG-IIc, a new type of congenital disorders of glycosylation, as a GDP-fucose transporter deficiency. Nature Gene. $2001 ; 28: 73-76$.

34. Lühn K, Wild MK, Eckhardt, et al. The gene defective in leukocyte adhesion deficiency II encodes a putative GDP-fucose transporter. Nature Genet. 2001; 28:69-72. [PubMed: 11326279]

35. Kuijpers TW, van de Vijver E, Weterman MAJ, et al. LAD-1/variant syndrome is caused by mutations in FERMT3. Blood. 2009; 113:4740-4746. [PubMed: 19064721]

36. Malinin NL, Zhang L, Choi J, et al. A point mutation in KINDLIN3 ablates activation of three integrin subfamilies in humans. Nature Med. 2009; 15:313-318. [PubMed: 19234460]

37. Svensson L, Howarth K, McDowall A, Patzak I, Evans R, Ussar S, Moser M, Metin A, Fried M, Tomlinson I, Hogg N. Leukocyte adhesion deficiency-III is caused by mutations in KINDLIN3 affecting integrin activation. Nature Med. 2009; 15:306-312. [PubMed: 19234463]

38. Williams DA, Tao W, Yang F, et al. Dominant negative mutation of the hematopoietic-specific Rho GTPase, Rac2, is associated with a human phagocyte immunodeficiency. Blood. 2000; 96:1646-1654. [PubMed: 10961859]

39. Walters KB, Green JM, Surfus JC, Yoo SK, Huttenlocher A. Live imaging of neutrophil motility in a zebrafish model of WHIM syndrome. Blood. 2010; 116:2803-2811. [PubMed: 20592249]

40. Haddy TB, Rana SR, Castro O. Benign ethnic neutropenia: what is a normal absolute neutrophil count? J Lab Clin Med. 1999; 133:15-22. [PubMed: 10385477]

41. Dale DC, Bonilla MA, Davis MW, et al. A randomized controlled phase III trial of recombinant human granulocyte colony-stimulating factor (filgrastim) for treatment of severe chronic neutropenia. Blood. 1993; 81:2496-2502. [PubMed: 8490166]

42. Liu F, Wu HY, Wesselschmidt R, et al. Impaired production and increased apoptosis of neutrophils in granulocyte colony-stimulating factor receptor-deficient mice. Immunity. 1995; 5:491-501. [PubMed: 8934575]

43. Plo I, Zhang Y, Le Couédic J-P, et al. An activating mutation in the CSF3R gene induces a hereditary chronic neutrophilia. J Exp Med. 2009; 206:1701-1707. [PubMed: 19620628]

44. Jung J, Bohn G, Allroth A, et al. Identification of a homozygous deletion in the AP3B1 gene causing Hermansky-Pudlak syndrome, type 2. Blood. 2006; 108:362-369. [PubMed: 16537806]

45. Elsner J, Roesler J, Emmendörffer A, et al. Abnormal regulation in the signal transduction in neutrophils from patients with severe congenital neutropenia: relation of impairs mobilization of cytosolic free calcium to altered chemotaxis, superoxide anion generation and F-actin content. Exp Hematol. 1993; 21:38-46. [PubMed: 7678087] 
46. Schäffer AA, Klein C. Genetic heterogeneity in severe congenital neutropenia: how many aberrant pathways can kill a neutrophil? Curr Opin Allergy Clin Immunol. 2007; 7:481-494. [PubMed: 17989524]

47. Okada Y, Kamatani Y, Takahashi A, et al. Common variations in PSMD3-CSF3 and PLCB4 are associated with neutrophil count. Hum Mol Genet. 2010; 19:2079-2085. [PubMed: 20172861]

48. Schmid JP, Ho C-H, Diana J, et al. A Griscelli syndrome type 2 murine model of hemophagocytic lymphohistiocytosis (HLH). Eur J Immunol. 2008; 38:3219-3225. [PubMed: 18991284]

49. Lieschke GJ, Grail D, Hodgson G, et al. Mice lacking granulocyte colony-stimulating factor have chronic neutropenia, granulocyte and macrophage progenitor cell deficiency, and impaired neutrophil mobilization. Blood. 1994; 84:1737-1746. [PubMed: 7521686]

50. Nagle DL, Karim MA, Woolf EA, et al. Identification and mutation analysis of the complete gene for Chediak-Higashi syndrome. Nature Genet. 1996; 14:307-311. [PubMed: 8896560]

51. Runkel F, Büssow H, Seburn KL, et al. Grey, a novel mutation in the murine Lyst gene, causes the beige phenotype by skipping of exon 25. Mamm Genome. 2006; 17:203-210. [PubMed: 16518687]

52. Belaaouaj A, McCarthy R, Baumann M, et al. Mice lacking neutrophil elastase reveal impaired host defense against gram negative bacterial sepsis. Nature Med. 1998; 4:615-618. [PubMed: 9585238]

53. Grenda DS, Johnson SE, Mayer JR, et al. Mice expressing a neutrophil elastase mutation derived from patients with severe congenital neutropenia have normal granulopoiesis. Blood. 2002; 100:3221-3228. [PubMed: 12384420]

54. Nanua S, Murakami M, Xia J, et al. Activation of the unfolded protein response is associated with impaired granulopoiesis in transgenic mice expressing mutant Elane. Blood. 2011; 117:35393537. [PubMed: 21285438]

55. Feng L, Seymour AB, Jiang S, et al. The $\beta 3 \mathrm{~A}$ subunit gene (Ap3b1) of the AP-3 adaptor complex is altered in the mouse hypopigmentation mutant pearl, a model for Hermansky-Pudlak syndrome and night blindness. Hum Molec Genet. 1999; 8:323-330. [PubMed: 9931340]

56. Blasius, Al; Arnold, CN.; Georgel, P., et al. Slc15a4, AP-3, and Hermansky-Pudlak syndrome proteins are required for Toll-like receptor signaling in plasmacytoid dendritic cells. Proc. Natl Acad Sci USA. 2010; 107:19973-19978. [PubMed: 21045126]

57. Wilson SM, Yip R, Swing DA, et al. A mutation in Rab27a causes the vesicle transport defects observed in ashen mice. Proc Natl Acad Sci USA. 2000; 97:7933-7938. [PubMed: 10859366]

58. Karsunky H, Zen H, Schmidt T, et al. Inflammatory reactions and severe neutropenia in mice lacking the transcriptional repressor Gfi1. Nature Genet. 2002; 30:295-300. [PubMed: 11810106]

59. Hock H, Hamblen MJ, Rooke HM, et al. Intrinsic requirement for zinc finger transcription factor Gfi-1 in neutrophil differentiation. Immunity. 2003; 18:109-120. [PubMed: 12530980]

60. Zarebski A, Velu CS, Baktula AM, et al. Mutations in growth factor independent-1 associated with human neutropenia block murine granulopoiesis through colony stimulating factor-1. Immunity. 2008; 28:370-380. [PubMed: 18328744]

61. Chen L-Y, Shieh J-J, Lin B, et al. Impaired glucose homeostasis, neutrophil trafficking and function in mice lacking the glucose-6-phosphate transporter. Hum Mol Genet. 2003; 12:25472558. [PubMed: 12925567]

62. Kim SY, Nguyen AD, Gao J-L, et al. Bone marrow-derived cells require a functional glucose 6phosphate transporter for normal myeloid functions. J Biol Chem. 2006; 39:28794-28801. [PubMed: 16891306]

63. Cheung YY, Kim SY, Yiu WH, et al. Impaired neutrophil activity and increased susceptibility to bacterial infection in mice lacking glucose-6-phosphatase- $\beta$. J Clin Invest. 2007; 117:784-793. [PubMed: 17318259]

64. Chao J-R, Parganas E, Boyd K, et al. Hax1-mediated processing of HtrA2 by Parl allows survival of lymphocytes and neurons. Nature. 2008; 452:98-102. [PubMed: 18288109]

65. Soustek MS, Falk DJ, Mah CS, et al. Characterization of a transgenic short hairpin RNA-induced murine model of tafazzin deficiency. Hum Gene Ther. 2011; 22:865-871. [PubMed: 21091282] 
66. Benson KF, Li F-Q, Person RE, et al. Mutations associated with neutropenia in dogs and humans disrupt intracellular transport of neutrophil elastase. Nature Genet. 2003; 35:90-96. [PubMed: 12897784]

67. Shearman JR, Wilton AN. A canine model of Cohen syndrome: Trapped Neutrophil Syndrome. BMC Genomics. 2011; 12:258. [PubMed: 21605373]

68. Klein C, Grudzien M, Appaswamy G, et al. Deficiency of HAX1 causes autosomal recessive severe congenital neutropenia (Kostmann disease). Nature Genet. 2007; 39:86-92. [PubMed: 17187068]

69. Boztug K, Appaswamy G, Ashikov A, et al. A syndrome with congenital neutropenia and mutations in G6PC3. N Engl J Med. 2009; 360:32-43. [PubMed: 19118303]

70. Kostmann R. Infantile genetic agranulocytosis (Agranulocystosis infantilis hereditaria): a new recessive lethal disease in man. Acta Paediatr. 1956; 45(Suppl.):1-78. [PubMed: 13313124]

71. Germeshausen M, Grudzien M, Zeidler C, et al. Novel HAX1 mutations in patients with severe congenital neutropenia reveal isoform-dependent genotype-phenotype associations. Blood. 2008; 111:4954-4957. [PubMed: 18337561]

72. Kolehmainen J, Black GCM, Saarinen A, et al. Cohen syndrome is caused by mutations in a novel gene, $\mathrm{COH} 1$, encoding a transmembrane protein with a presumed role in vesicle-mediated sorting and intracellular protein transport. Am J Hum Genet. 2003; 72:1359-1369. [PubMed: 12730828]

73. Cohen MM Jr, Hall BD, Smith DW, et al. A new syndrome with hypotonia, obesity, mental deficiency, and facial, oral, ocular and limb anomalies. J Pediatr. 1973; 83:280-284. [PubMed: 4717588]

74. Norio R, Raitta C, Lindahl E. Further delineation of the Cohen syndrome; report on chorioretinal dystrophy, leukopenia and consanguinity. Clin Genet. 1984; 25:1-14. [PubMed: 6705238]

75. Person RE, Li F-Q, Duan Z, Benson KF, et al. Mutations in proto-oncogene GFI1 cause human neutropenia and target ELA2. Nature Genet. 2003; 34:308-312. [PubMed: 12778173]

76. Hernandez PA, Gorlin RJ, Lukens JN, et al. Mutations in the chemokine receptor gene CXCR4 are associated with WHIM syndrome, a combined immunodeficiency disease. Nature Genet. 2003; 34:70-74. [PubMed: 12692554]

77. Ridanpää M, van Eenennaam H, Pelin K, et al. Mutations in the RNA component of RNase MRP cause a pleiotropic human disease, cartilage-hair hypoplasia. Cell. 2001; 104:195-203. [PubMed: 11207361]

78. Volpi L, Roversi G, Colombo EA, et al. Targeted next-generation sequencing appoints C16orf57 as Clericuzio-type poikiloderma with neutropenia gene. Am J Hum Genet. 2010; 86:72-76. [PubMed: 20004881]

79. Hamasaki A, Sendo F, Nakayama K, et al. Accelerated neutrophil apoptosis in mice lacking A1-a, a subtype of the bcl-2-related A1 gene. J Exp Med. 1998; 188:1985-1992. [PubMed: 9841913]

80. Dzhagalov I, St. John A, He Y-W. The antiapoptotic protein Mcl-1 is essential for the survival of neutrophils but not macrophages. Blood. 2007; 109:1620-1626. [PubMed: 17062731]

81. Lagresle-Peyrou C, Six EM, Picard C, et al. Human adenylate kinase 2 deficiency causes a profound hematopoietic defect associated with sensorineural deafness. Nature Genet. 2009; 41:106-111. [PubMed: 19043416]

82. Pannicke U, Honig M, Hess I, et al. Reticular dysgenesis (aleukocytosis) is caused by mutations in the gene encoding mitochondrial adenylate kinase 2. Nature Genet. 2009; 41:101-105. [PubMed: 19043417]

83. Hart TC, Hart PS, Bowden DW, et al. Mutations of the cathepsin C gene are responsible for Papillon-Lefèvre syndrome. J Med Genet. 1999; 36:881-887. [PubMed: 10593994]

84. Toomes C, James J, Wood AJ, et al. Loss-of-function mutations in the cathepsin C gene result in periodontal disease and palmoplantar keratosis. Nature Genet. 1999; 23:421-424. [PubMed: 10581027]

85. Pham CTN, Ley TJ. Dipeptidyl peptidase I is required for the processing and activation of granzymes A and B in vivo. Proc Natl Acad Sci USA. 1999; 96:8627-8632. [PubMed: 10411926]

86. Pagano MB, Bartoli MA, Ennis TL, et al. Critical role of dipeptidyl peptidase I in neutrophil recruitment during the development of experimental abdominal aortic aneurysms. Proc Natl Acad Sci USA. 2007; 104:2855-2860. [PubMed: 17301245] 
87. Huang C-K, Zhan L, Hannigan MO, et al. P47phox-deficient NADPH oxiase defect in neutrophils of diabetic mouse strains, C57BL/6J-m db/ db and db/ + J Leukoc Biol. 2000; 67:210-215. [PubMed: 10670582]

88. Hultqvist M, Oloffson P, Holmberg J, et al. Enhanced autoimmunity, arthritis, and encephalomyelitis in mice with a reduced oxidative burts due to a mutation in the Ncf1 gene. Proc Natl Acad Sci USA. 2004; 101:12646-12651. [PubMed: 15310853]

89. Vethanayagam RR, Almyroudis NG, Grimm MJ, et al. Role of NADPH oxidase versus neutrophil proteases in antimicrobial host defense. PLoS ONE. 2011; 6:e28149. [PubMed: 22163282]

90. Colucci-Guyon E, Tinevez J-Y, Renshaw SA, et al. Strategies of professional phagocytes in vivo: unlike macrophages, neutrophils engulf only surface-assoicated microbes. J Cell Sci. 2011; 124:3053-3059. [PubMed: 21868367]

91. Khuchua Z, Ye Z, Batts L, Strauss AW. A zebrafish mdel of human Barth syndrome reveals the essential role of tafazzin in cardiac development and function. Circulation Res. 2006; 99:201-208. [PubMed: 16794186]

92. Jacob CO, Eisenstein M, Dinauer MC, et al. Lupus-associated causal mutation in neutrophil cytosolic factor 2 (NCF2) brings unique insights to the structure and function of NADPH oxidase. Proc Natl Acad Sci USA. 2012; 109:E59-E67. [PubMed: 22203994]

93. Muise AM, Xu W, Guo C-H, et al. NADPH oxidase complex and IBD candidate gene studies: identification of a rare variant in NCF2 that results in reduced binding to RAC2. Gut. 2012; 61:1028-1035. [PubMed: 21900546]

94. Beutler B, Moresco EM. The forward genetic dissection of afferent innate immunity. Curr Top Microbiol Immunol. 2008; 321:3-26. [PubMed: 18727485]

95. Cook MC, Vinuesa CG, Goodnow CC. ENU-mutagenesis: insight into immune function and pathology. Curr Opin Immunol. 2006; 18:627-633. [PubMed: 16889948]

96. Soewarto D, Fella C, Teubner A, et al. The large-scale Munich ENU-mouse-mutagenesis screen. Mamm Genome. 2000; 11:507-510. [PubMed: 10886013]

97. Xia J, Bolyard AA, Rodger E, Stein S, et al. Prevalence of mutations in ELANE, GFI1, HAX1, SBDS, WAS, and G6PC3 in patients with severe congenital neutropenia. Br J Haematol. 2009; 147:535-542. [PubMed: 19775295]

98. Smith BN, Evans C, Ali A, et al. Phenotypic heterogeneity and evidence of a founder effect associated with G6PC3 mutations in patients with severe congenital neutropenia. Br J Haematol. 2012

99. Rosenberg PS, Alter BP, Bolyard AA, et al. The incidence of leukemia and mortality from sepsis in patients with severe congenital neutropenia receiving long-term G-CSF therapy. Blood. 2006; 107:4628-4635. [PubMed: 16497969]

100. Mardiney M III, Jackson SH, Spratt SK, et al. Enhanced host defense after gene transfer in the murine p47phox-deficient model of chronic granulomatous disease. Blood. 1997; 89:2268-2275. [PubMed: 9116268]

101. Jackson SH, Miller GF, Segal BH, Mardiney M 3rd, et al. IFN-gamma is effective in reducing infections in the mouse model of chronic granulomatous disease (CGD). J Interferon Cytokine Res. 2001; 21:567-573. [PubMed: 11559434]

102. Fernandez-Boyanapalli R, McPhillips KA, Frasch SC, et al. Impaired phagocytosis of apoptotic cells by macrophages in chronic granulomatous disease is reversed by IFN- $\gamma$ in a nitric oxidedependent manner. J Immunol. 2010; 185:4030-4041. [PubMed: 20805415]

103. Laine DI, Busch-Petersen J. Inhibitors of cathepsin C (dipeptidyl peptidase I). Expert Opin Ther Pat. 2010; 20:497-506. [PubMed: 20205619] 


\section{Key Points}

- Human granulocyte diseases are mostly monogenic diseases affecting neutrophils, not eosinophils and basophils.

- Most monogenic neutrophil diseases are recessive or X-linked and can be classified as chronic granulomatous disease (CGD), leukocyte adhesion deficiency (LAD), or severe congenital neutropenia (SCN). Each of these can be caused by mutations in different genes.

- For CGD and LAD, mice bred to lack the orthologous gene mutated in each human form usually have a phenotype similar to that of the human patients. In contrast, for SCN, there is a striking discrepancy between the phenotypes of model mice and human patients.

- Not all animal models are mouse models. For some neutrophil diseases, there are naturally occurring models in dogs or cows. The construction of zebrafish models is an emerging trend in neutrophil diseases.

- Opportunities to characterize new monogenic forms of SCN, to generate new mouse models by random mutagenesis, to engineer new zebrafish models, and to use animal models in the exploration of new treatments should occupy researchers studying animal models of neutrophil diseases for many years to come. 


\section{Table 1}

Human genes mutated in chronic granulomatous disease and corresponding mouse models with a mutation of the orthologous gene. Reference numbers are in the third and fourth columns.

\begin{tabular}{|l|l|l|l|}
\hline Protein & Gene & Human mutation ref. & Mouse model ref. \\
\hline p22 & CYBA & 11 & 12 \\
\hline p40 & NCF4 & 13 & 14 \\
\hline p47 & NCF1 & 15 & 16 \\
\hline p67 & NCF2 & 17 & 18 \\
\hline gp91 & CYBB & 19 & 20 \\
\hline
\end{tabular}


Table 2

Animal models for different types of leukocyte adhesion deficiency (LAD).

\begin{tabular}{|l|l|l|l|l|}
\hline Disease & Human Gene & Model Organism & Mutation & Reference(s) \\
\hline LAD I & ITGB2 (CD18) & Mouse & hypomorphic & 24 \\
\hline LAD I & ITGB2 (CD18) & Mouse & Null & 25 \\
\hline LAD I & ITGB2 (CD18) & Cows & D128G & 26 \\
\hline LAD II & SLC35C1 & Mice & Null & 27 \\
\hline LAD III & FERMT3 (kindlin) & Mice & null and null only in chimeras and neutrophils & 28,29 \\
\hline RAC2 disease & RAC2 (D57N mutation) & Mice & Null & 30,31 \\
\hline RAC2 disease & RAC2 (D57N mutation) & Zebrafish & Morpholino or D57N & 21 \\
\hline
\end{tabular}




\section{Table 3}

Mouse models mutating the orthologs of genes mutated or expected to be mutated in human SCN. For all rows, the symbol of the mouse orthologous gene is identical to the human symbol except that letters after the first are upper case for human become lower case for mouse (e.g., human $H A X 1$ is orthologous to mouse Hax 1). Unlike CGD and LAD, the mouse models for SCN are not necessarily neutropenic, so we note the phenotype correspondence in the rightmost column. In several cases of syndromic phenotypes, the papers describing the mice make no mention of neutrophil counts. The genes are listed in the order of the first publication year for a mouse model related to each gene, with ties broken arbitrarily.

\begin{tabular}{|c|c|c|c|c|}
\hline Gene & $\begin{array}{l}\text { Human } \\
\text { mutations/inheritance }\end{array}$ & $\begin{array}{l}\text { Mouse } \\
\text { mutation }\end{array}$ & $\begin{array}{l}\text { Reference for } \\
\text { mouse model }\end{array}$ & $\begin{array}{l}\text { Are mice } \\
\text { neutropenic? }\end{array}$ \\
\hline CSF3 & None? & Null & 49 & Yes \\
\hline CSF3R & Somatic mutations only(?) & Null & 42 & Yes \\
\hline$L Y S T$ & Biallelic/recessive & Null? (beige) & 50 & No? \\
\hline$L Y S T$ & Biallelic/recessive & Skip exon 25 (grey) & 51 & No? \\
\hline$E L A 2$ & Heterozygous/dominant & Null & 52 & $\begin{array}{l}\text { No, but neutrophil function } \\
\text { is aberrant }\end{array}$ \\
\hline$E L A 2$ & Heterozygous mutations/dominant & V72M heterozygous knock-in & 53 & No \\
\hline$E L A 2$ & Heterozygous mutations/dominant & $\begin{array}{l}\text { G193X heterozygous or homozygous } \\
\text { knock-in }\end{array}$ & 54 & $\begin{array}{l}\text { No, but } \mathrm{SCN} \text { can be induced } \\
\text { by a proteasome inhibitor }\end{array}$ \\
\hline$A P 3 B 1$ & Biallelic/recessive & Null (pearl) & 55 & No \\
\hline$A P 3 B 1$ & Biallelic/recessive & Null (bullet gray) & 56 & No \\
\hline$R A B 27 A$ & Biallelic/recessive & Null (ashen) & 57 & Yes, after LCMV infection ${ }^{48}$ \\
\hline GFI1 & Heterozygous/dominant negative & Null & 58,59 & Yes \\
\hline GFI1 & Heterozygous/dominant negative & $\begin{array}{l}\text { N382S retrovirally induced in cultured } \\
\text { cells }\end{array}$ & 60 & $\begin{array}{l}\text { Granulopoiesis blocked, but } \\
\text { no ANC measured since this } \\
\text { model is in vitro only }\end{array}$ \\
\hline SLC37A4 & Biallelic/recessive & Null & 61 & Yes \\
\hline SLC37A4 & Biallelic/recessive & Null + cell transfer & 62 & Yes \\
\hline G6PC3 & Biallelic/recessive & Null & 63 & Yes \\
\hline$H A X 1$ & Biallelic/recessive & Null & 64 & No \\
\hline$T A Z$ & $\mathrm{X}$-linked & Knock down by RNAi & 65 & Some animals \\
\hline
\end{tabular}


Table 4

Non-mouse models for SCN in which neutrophils were tested and shown to be deficient.

\begin{tabular}{|l|l|l|l|l|}
\hline Organism & Gene & Inheritance & Mutation & Reference \\
\hline Dog (collie) & $A P 3 B 1$ & Recessive & Null & 66 \\
\hline Zebrafish & $C X C R 4$ & Dominant & Transgenic expression of human mutation & 39 \\
\hline Dog (border collie) & $V P S 13 B$ & Recessive & Four nucleotide deletion; may not affect all transcripts & 67 \\
\hline
\end{tabular}

